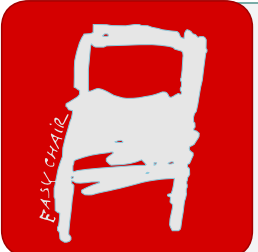

EPiC Series in Health Sciences

Volume 2, 2018, Pages 208-218

CAOS 2018. The 18th Annual Meeting of the International Society for Computer Assisted Orthopaedic Surgery

\title{
The accuracy and safety of patient-specific 3D printing screws insertion templates based on external fixation for pelvic screw insertion
}

\author{
Xing-Guang Tao MD, Hui Liang MSc, Fu-Gen Pan MD and Kai-Hua Zhou MD* \\ Qingpu Branch of Zhongshan Hospital of Fudan University, Shanghai, China
}

1983216145@sohu.com

\section{Introduction}

The incidence of pelvic fracture accounts for $2-8 \%$ of all fractures of the human body (1). It usually results from massive and commonly life-threatening external forces. This sort of high-energy trauma results in predominantly unstable pelvic injuries. For unstable pelvic fractures (Tile type B and type C) in an emergency setting, we commonly use external fixation for damage control $(2,3)$. When the vital signs of the patients are stable, the final internal fixation is performed. As open reduction and internal fixation with plates may lead to secondary damage, considerably more intraoperative blood loss and iatrogenic injury of the nerve and blood vessels, most scholars $(4,5)$ recommend percutaneous screw fixation to treat the pelvic fractures if possible. However, in the traditional minimally invasive percutaneous screw fixation surgery, C-arm fluoroscopy is used more frequently to make sure the screw is in the ideal position, which obviously increases the operation time and the X-ray exposure to the patients and surgeon. With the development of the two-dimensional (2D) and 
three-dimensional (3D) navigation system, the minimally invasive percutaneous screw technique is now becoming a safe, accurate and fairly quick method to treat the pelvic ring fracture (6). However, the navigation system is very expensive, and not every hospital can be equipped with it. Now, with the continuous advance of the 3D printing technique, we can use the patient-specific screw guide template, instead of the navigation system, to aid minimally invasive percutaneous surgery.

3D printing is a kind of novel industrial manufacturing technique which integrates laser rapid prototyping technology, polymer material and three-dimensional CT technology (7). Currently, the main application of 3D printing in orthopedics is in three areas: preoperative planning, surgical guide template and implant printing. The surgical guide template is based on the patient's individual data, which has been successfully used in arthroplasty, spine and osteotomy surgery (8). Traditionally, in order to obtain a good match, we should put the 3D printing template close to the bone surface which leads to large dissection of the soft tissue (9-11). However, in the pelvic area, the soft tissue is thicker and adjacent to important vessels and nerves, thus the traditional 3D printer template technique is mostly unsuitable. For the unstable pelvic fractures, we usually need external fixation first to achieve damage control during the first stage. Based on this condition, we design the patient-specific screw guide template based on the external fixation to insert the antegrade pubic rami screw and sacroiliac screw using the minimally invasive percutaneous technique without dissection of the soft tissue. The purpose of this study is to investigate the feasibility and accuracy of this technique.

Materials and Methods

Before this study, we obtained approvals by the ethics review committee of the Qingpu branch of Zhongshan hospital of Fudan University and written informed consent was obtained from the patient

From January to June in 2017, 6 patients with Tile B type pelvic fractures were involved in this study, including 5 males and 1 female. The average age was 52.3 years (range 23 78). According to the Tile classification, there were two type B1, three type B2.1, one type B3. All the patients 
underwent external fixation to achieve damage control and performed a closed reduction ati the same time in the emergency room during the first stage. The external fixation (Trauson, China) was performed with a Carefix composite scaffold, which consists of 6-mm diameterstainless steel spicules, an 8-mm diameter aluminum alloybar material and an 8-mm diametercarbon fiber/PEEK unilateral connecting rod.

\section{Preparation of template}

The patients with external fixation were examined by 64 spiral computed tomography (CT) scanner(GE, Boston, MA, USA) with $5 \mathrm{~mm}$ slice thickness before operation. The images were stored in DICOM format and analyzed by the Mimics 10.01 . The trajectory and the depth of the screw were designed in 3D format. We adopted $6.5 \mathrm{~mm}$ as the optimal diameter of the screw. A virtual cylindrical implant with a diameter of $6.5 \mathrm{~mm}$ was placed in the axis of the pubic rim and $\mathrm{S} 1$ body. The direction was adjusted until the optimal screw path was found using the reverse engineering technique to make sure that the virtual cylindrical implant was totally in the bony structure and did not penetrate the acetabular and pubis rim cortex or the sacral foramen. In addition, 2D images of the pelvis in transverse, coronal and sagittal planes were observed to confirm that the virtual cylindrical implant was intraosseous. We also measured the length of the cylindrical implant. After the screw entry point and the tip of the screw were determined, the data were saved and derived in the STL format.

The above data were input into the Geomagic studio 2012 software. Then, the patient-specific screw guide template and 3D model of the pelvis were designed . Subsequently, just as designed, the patient-specific template and the 3D bone model were made using a 3D printing system (1 iantai RS6000) with photosensitive resin material (Fig 1).

\section{Surgery}

The engagement of the screw guide template with the $3 \mathrm{D}$ model and screw simulation was confirmed before surgery. Then, the template was sterilized by ethylene oxide. After general 
anesthesia, the patient was in supine position and the assistant connected the $3 \mathrm{D}$ printing template to the external fixation. A $1.5 \mathrm{~cm}$ incision was made according to the direction of the guide hole on the template. The assistant used one hand to stabilize the template and external fixation. The surgeon drilled $2.5 \mathrm{~mm}$ relatively rigid $\mathrm{K}$-wires along the guide hole to the pubic rim and $\mathrm{S} 1$ body. The $\mathrm{C}$-arm was used to confirm the position of the K-wire during the insertion process. When the K-wire was in the right position, the template was removed and the length of the screw was measured. Appropriated $6.5 \mathrm{~mm}$ cannulated screws (AO, Synthes, Stratec Medical, Oberdorf, Switzerland) were inserted along the $\mathrm{K}$-wires which was the similar length as in the preoperative plan. The $\mathrm{C}$-arm was used again to assure the position and length of the screw. Finally, the K-wire was removed and the incision was sutured (Fig2)

\section{Postperative assessment of screw placement}

Radiographic imaging in the form of X-rays and CT scan was repeated after surgery and checked for fracture reduction and hardware placement. We also calculated the screw deviation from the planned trajectory.

\section{Results}

Totally 10 screws were inserted, including 5 pubis screws and 5 sacroiliac screws. The average surgical time for pubis screw was $12.7 \mathrm{~min} / \mathrm{screw}$, and $9.2 \mathrm{~min} / \mathrm{screw}$ for sacroiliac screw. The average time of X-ray exposure was $13.3 \pm 3.6 \mathrm{~s} / \mathrm{screw}$ for public screw and $9.6 \pm 4.5 \mathrm{~s} / \mathrm{screw}$ for sacroiliac screw. All the screws were inserted in the trajectory as preoperative design and the screw axis deviation was $1.60 \pm 0.2 \mathrm{~mm}$ and $2 \pm 0.3$ degrees angle deviation by preoperative and postoperative CT verification. Blood loss during the surgery was minimal (12.2ml/screw). Vascular of neurologic complications or injuries did not happen. And no infection, broken screws or screw pullout occurred. 


\section{Discussion}

Surgical treatment of unstable pelvic fracture (Tile type B and type C) has become the standard therapeutic approach but remains a great challenge for surgeons. The objective of the treatment is to stabilize the pelvic ring. For the treatment of unstable pelvic fracture with both anterior and posterior ring injury, most surgeons recommend external fixation to achieve damage control during the first stage, and internal fixation can be performed later when the vital signs of the patient become stable. For the anterior column of the acetabulum and superior pubic ramus fractures, we previously used the long ilio-inguinal incision, but now we mostly use the Stoppa's approach with reconstruction plates. However, all the traditional techniques of open reduction and internal fixation require extensive surgical exposure of the deep structures of the pelvis, which can slow or prevent wound healing, damage major vessels or nerves and increased the incidence of infection up to $25 \%(12,13)$. Some surgeons suggested using a subcutaneous internal anterior screw-rod fixation to avoid the disadvantages of the external implant with low rates of wound infections (14). However, it was associated with new complications, like injury of the femoral lateral cutaneous nerve and femoral nerve or heterotopic ossifications (15). Accordingly, dissection and exposure of the soft tissue is associated with these complications. If possible, a percutaneous screw is preferred. Besides, it can also stabilize the pelvic fracture while needing less surgical exposure. Biomechanical tests showed that lag screw fixation was at least as effective as plate fixation (16-19). The use of percutaneous screw is the trend for minimally invasive surgery for pelvic fractures.

Aside from the advantages of the percutaneous screw, it is a difficult technique that has potential risks of vessel and nerve damage, acetabulum penetration and the surgeon team is exposed to high levels of fluoroscopic times $(20,21,22)$. Thus, the percutaneous screw fixation is demanding and requires the surgeon to have detailed anatomic knowledge and extensive surgical experience. The fluoro-navigation technique could become a safe, accurate and fairly quick method for the insertion of 
percutaneous screws. However, the cost of the navigation equipment is too high, and most hospitals cannot generally be equipped with it. On the other hand, we have to insert a titanium tracker preoperatively for registering, which also add another incision to the patient $(23,24)$. To insert the screw in the ideal position and have the same effect as the fluoro-navigation surgery with low cost, we designed a patient-specific screw guide template based on the external fixation to insert the pubis rim screw by $3 \mathrm{D}$ printing.

Patient-specific screw guide template is based on the patient individual CT data, computational software, reverse engineering and $3 \mathrm{D}$ printing technique. The template can guide us to the entry point and the direction of the guide pin and screw, which can make the operation much more accurate, convenient and safe. However, the normal template needs bone surfaces to engage each other. Accordingly, we still have to dissect the soft tissue and expose the bone surface during the operation. Some surgeons have used this method to insert the screw to treat pelvic fracture, but the dissection of soft tissue lacks the advantage of minimally invasive percutaneous screw technique $(25,26)$.

Our specific template is based on the external fixation. The advantage is that the screw guide template is engaged with the external fixation not the bone surface, thus we should not need to dissect the soft tissue, just like in the fluoro-navigation surgery. In this case, the template design is used at the same time during the resuscitation phase, so there is no delay to the diagnosis and treatment of this patient with this technique. There is small displacement both in the anterior and posterior wing of the pelvic, so we decided to use percutaneous screws to fix the fractures. The design principle of the template is similar to other bone surface matching templates, based on the external fixation shape to design the guide and bridging rods. The bridging rod should match at least two of the rods of the external fixation and the inner radius of curvature is the same as the radius of the external radius. The two template rods are preferably not parallel, so there is only one location to place the template. The design of the guide hole is based on the $\mathrm{CT}$ data of the patient to choose the best trajectory. The inner 
diameter of the guide hole is slightly larger than the diameter of the $\mathrm{K}$-wire in order to pass the K-wire through easily. We should also consider the soft tissue thickness according to the CT 3D reconstruction because the template is close to the skin instead of close to the bone surface. We currently use photosensitive hard resin material to print the template. However, the material can be deformed with a certain force. To ensure accuracy, the guide hole should be close to the bride rod and entry point. The K-wire should also be gently placed to avoid the crushing of the template. The assistant controlled the template when inserting the K-wire to decrease the deformation of the template and control its position. According to this design principle, the template can also be used in the other percutaneous screws with closed reduction and external fixation in the treatment of pelvic fractures, such as acetabular column screws, iliac wing screws, etc.

Although according to the result of this study the newly designed approach offers accuracy and safety, there is still $1.1 \pm 0.6 \mathrm{~mm}$ deviation and $2.5^{\circ} \pm 0.4^{\circ}$ in trajectory compared to the preoperative plan. This deviation may be caused by factors, such as the CT slice thickness, data conversion due to slight difference between the software model and the actual pelvic bone, the method used to deal with the CT data of the external fixation, the template size, the print material, the stability of the template in the operation and the elastic modulus of the template, etc. Therefore, we should minimize the deviation factors. We suggest that: (1) the thickness of the CT scan does not exceed $1 \mathrm{~mm}$ to reduce the distortion of the data conversion and 3D reconstruction model; (2) the chosen template material should be a larger elastic modulus, resistant to high temperature, not easily deformed and easy to disinfect and operate; (3) more than two the external fixation rods should be chosen to increase the fitness and stability of the template;(4) when the K-wire is drilled through the guide hole, operation should be soft and moderate, the K-wire should be drilled along the guide hole. We do not compress the template, which can cause the deformation of the template to reduce the accuracy.

Conclusion 
The patient-specific screw guide template based on external fixation can be used to accurately and safely insert the screw with a very small incision. This technique can significantly reduce the operation time and radiation exposure to the patient and surgeon team. The patient-specific screw guide template also can help the resident with less experience to insert the percutaneous screw more effectively through good preparation. With the further maturity of the technology and clinical verification, many more orthopedic surgeons of the basic hospitals maybe choose it to treat patients. The application of this technique will play an important role in the future clinical practice.

Reference:

1.Rommens PM. Is there a role for percutaneous pelvic and acetabular reconstruction? Injury, 2007, 38(4): 463-477

2. Wong JM, Bucknill A. Fractures of the pelvic ring. Injury. 2013, 48(4):795

3. Zhou KH, Luo CF, Chen N, Hu CF, Pan FG(2016) Minimally invasive surgery under fluoronavigation foranterior pelvic ring fractures. Indian J Orthop 2016,50:250-255

4. Huang H, Hsieh MF, Zhang G, Ouyang H, Zeng C, Yan B, Xu J, Yang Y, Wu Z, Huang W(2015)Improved accuracy of 3D-printed navigational template during complicated tibial plateau fracture surgery. Australasian physical \& engineering sciences in medicine, Mar;38(1):109-117.

5. Omori S, Murase T, Kataoka T, Kawanishi Y, Oura K, Miyake J, Tanaka H, Yoshikawa $\mathrm{H}(2014)$ Three-dimensional corrective osteotomy using a patient-specific osteotomy guide and bone plate based on a computer simulation system: accuracy analysis in a cadaver study. The international journal of medical robotics + computer assisted surgery : MRCAS, Jun;10(2):196-202. 
6. Kaneyama S, Sugawara T, Sumi M, Higashiyama N, Takabatake M, Mizoi K(2014)A novel screw guiding method with a screw guide template system for posterior C-2 fixation: clinical article. Journal of neurosurgery Spine, Aug;21(2):231-238

7.Malancea RI, Malancea R, Veliceasa B, Chirila D, Alexa O (2016) Treatment of pubic disjunction in young-burguess type II and III pelvic ring fractures. Revista medico-chirurgicala a Societatii de Medici si Naturalisti din Iasi., Jan-Mar;120(1):130-136.

8.Balbachevsky D, Belloti JC, Doca DG, Jannarelli B, Junior JA, Fernandes HJ, Dos Reis FB (2014) Treatment of pelvic fractures - a national survey. Injury, Suppl 5:S46-S51

9.Kellam JF, McMurtry RY, Paley D, Tile M(1987)The unstable pelvic fracture. Operative treatment. Orthop Clin North Am,18(1):25-41.

10.Routt ML Jr, Simonian PT, Swiontkowski MF(1997)Stabilization ofpelvic ring disruptions. Orthop Clin North Am,28(3):369-88.

11. Scheyerer MJ, Zimmermann SM, Osterhoff G, Tiziani S, Simmen HP, Wanner GA, Werner $\mathrm{CM}(2014)$ Anterior subcutaneous internal fixation for treatment of unstable pelvic fractures. BMC research notes, $8 ; 7: 133$

12.Gardner MJ, Mehta S, Mirza A, Ricci WM(2011) Anterior pelvic reduction andfixation using a subcutaneous internal fixator. J Orthop Trauma,26(5):314-321

13.Chang JK, Gill SS, Zura RD, Krause WR, Wang GJ(2001) Comparative strength of three methods of fixation of transverseacetabular fractures. Clin Orthop Relat Res,392:433-441

14. Shazar N, Brumback RJ, Novak VP, Belkoff SM(1998)Biomechanical evaluation of transverse acetabular fracture fixation. Clin Orthop Relat Res,352:215-222

15.Lazaro Gonzalvez A, Martinez Reina J, Cano Luis P, Jimenez Baquero J, Sueiro Fernandez J, Giraldez Sanchez MA(2016) Is cannulated-screw fixation an alternative to plate osteosynthesis in open book fractures? A biomechanical analysis. Injury, Sep;47 Suppl 3:S72-s77. 
16. Yu KH, Hong JJ, Guo XS, Zhou DS(2015) Comparison of reconstruction plate screw fixation and percutaneous cannulated screw fixation in treatment of Tile B1 type pubic symphysis diastasis: a finite element analysis and 10-year clinical experience. Journal of orthopaedic surgery and research,Sep 22;10:151..

17 Dzupa V, Nemec J, Pavlicko Z, Labos M, Sram J, Taller S, Baca V(2016)Minimal Invasive Fixation of Pelvic Injury: CT-Study of the Pelvic Bone Dimensions. Acta chirurgiae orthopaedicae et traumatologiae Cechoslovaca,83(3):147-154.

18.Hinsche AF, Giannoudis PV, Smith RM(2002) Fluoroscopy-basedmultiplanar image guidance for insertion of sacroiliac screws.Clin Orthop Relat Res ,395:135-144

19.Norris BL, Hahn DH, Bosse MJ, Kellam JF, Sims SH(1999)Intraoperativefluoroscopy to evaluate fracture reduction and hardwareplacement during acetabular surgery. J Orthop Trauma, $13(6): 414-417$

20. Docquier PL, Paul L, Cartiaux O, Banse X(2009)Registration accuracy in computer-assisted pelvic surgery. Computer aided surgery : official journal of the International Society for Computer Aided Surgery, 14(1-3):37-44

21. Kang HG, Cho CN, Kim KG(2014)Percutaneous navigation surgery of osteoid osteoma of the femur neck. Minimally invasive therapy \& allied technologies : MITAT : official journal of the Society for Minimally Invasive Therapy,23(1):58-62

22.Chen H, Wang G, Li R, Sun Y, Wang F, Zhao H, Zhang P, Zhang X(2016) A novel navigation template for fixation of acetabular posterior column fractures with antegrade lag screws: design and application. International orthopaedics,40(4):827-834.

23. Chen B, Zhang Y, Xiao S, Gu P, Lin X(2012)Personalized image-based templates for iliosacral screw insertions: a pilot study. The international journal of medical robotics + computer assisted surgery : MRCASa,Dec;8(4):476-482 
Fig1, A virtual cylindrical implant with a diameter of $6.5 \mathrm{~mm}$ was placed in the axis of the pubic rim and S1 body and the patient-specific screw guide template was designed
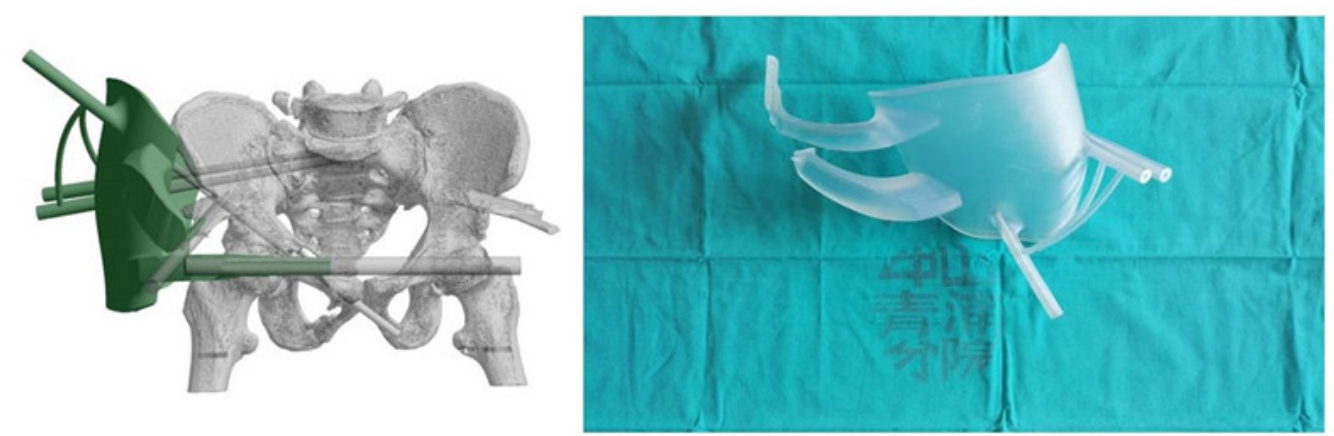

Fig2.three cannulated screws were inserted according to the Patient-specific screw guide template and postoperative $\mathrm{X}$-ray
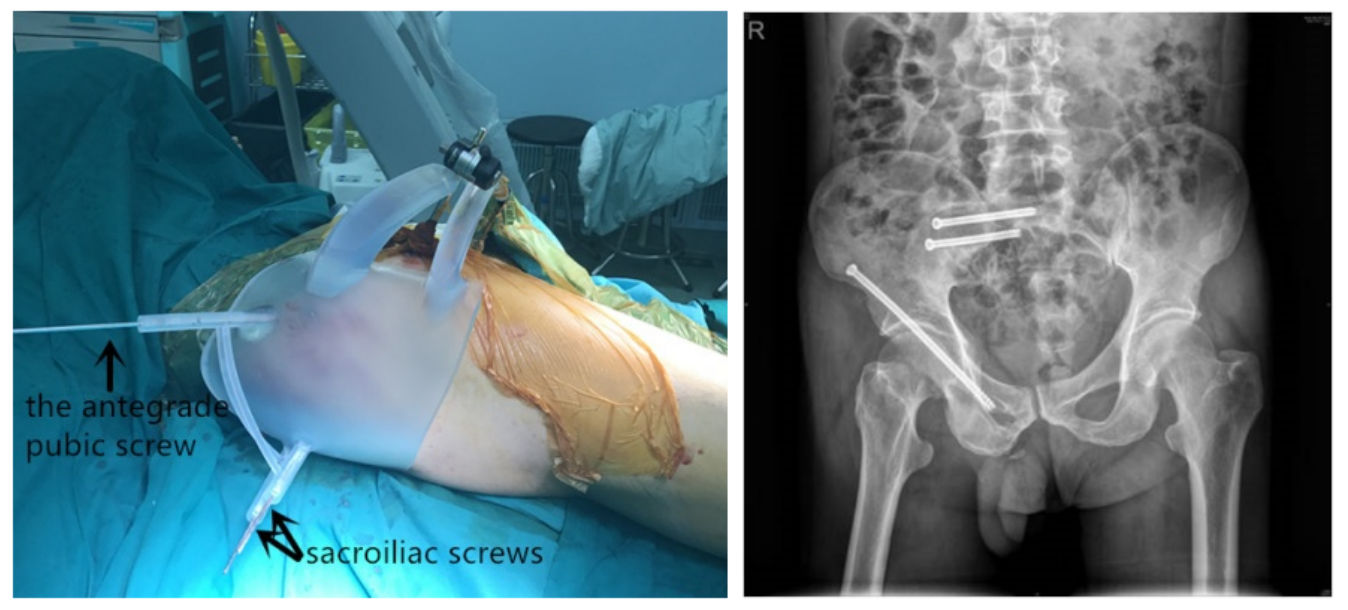